\title{
VIIRS THERMAL EMISSIVE BANDS L1B CALIBRATION UNCERTAINTY
}

\author{
Kwofu V. Chiang ${ }^{1}$, Jeff McIntire ${ }^{1}$, and Xiaoxiong Xiong ${ }^{2}$ \\ ${ }^{1}$ Science Systems and Applications, Inc., 10210 Greenbelt Road, Lanham, MD 20706, USA. \\ ${ }^{2}$ Sciences and Exploration Directorate, NASA/GSFC, Greenbelt, MD 20771, USA.
}

\begin{abstract}
The Visible Infrared Imaging Radiometer Suite (VIIRS) is a key instrument on-board the Suomi National Polar-orbiting Partnership (S-NPP) spacecraft. The S-NPP launched in October 2011 and it has been collecting valuable Earth science data with VIIRS and four other instruments for more than five years. The VIIRS Characterization Support Team (VCST) of the National Aeronautics and Space Administration (NASA) Science Investigator-led Processing Systems (SIPS) is designed to support the VIIRS sensor prelaunch geometric and radiometric characterization and to access on-orbit long-term Level-1B (L1B) calibration and performance. This paper reviews the VIIRS thermal emissive bands (TEB), covering wavelengths from 3.7 to $12.0 \mu \mathrm{m}$, L1B radiometric calibration algorithms and presents the calibration uncertainty methodology which will be implanted in the L1B processing software. Discussions will be focused on the key uncertainty parameters and the application in L1B.
\end{abstract}

Index Terms - VIIRS, S-NPP, TEB, Uncertainty, VCST

\section{INTRODUCTION}

The Visible Infrared Imaging Radiometer Suite (VIIRS) is currently operating on Suomi National Polar-orbiting Partnership (S-NPP) spacecraft. The VIIRS Flight Unit 1 onboard the S-NPP was launched on October 28, 2011. VIIRS extends and improves the measurements by its predecessors the Advanced Very High Resolution Radiometer (AVHRR) and the Moderate Resolution Imaging Spectroradiometer (MODIS) in many ways. Since its launch, the S-NPP VIIRS has generated many Sensor Data Record (SDR) and Environmental Data Record (EDR) covering land, ocean, and atmosphere that are critical to long-term Earth science research about climate change. VIIRS will also be included in the Joint Polar Satellite Systems (JPSS) mission, a NOAA/NASA program, to continue providing high quality observations day and night. The follow-on VIIRS instrument Flight Unit 2 onboard the JPSS-1 spacecraft is schedule to be launched later in 2017. Similar to MODIS design, VIIRS uses an optical scanning imaging system to observe the top of atmosphere (TOA) properties at an altitude of $824 \mathrm{~km}$ in a Sun-synchronous polar orbit. The observations provide complete Earth coverage every two days with a 16-day repeat cycle. Instead of using a large double-sided scan mirror at the nadir port like MODIS, the VIIRS uses a Rotating Telescope Assembly (RTA) coupled with a half-speed Half Angle Mirror (HAM) to achieve the wide scan angle $\pm 56^{\circ}$ off nadir, corresponding to a swath of about $3000 \mathrm{~km}$. VIIRS has three types of bands at different spatial resolution, including 16 moderate (M) resolution bands (M1-M16; each has 16 detectors in an array along the track direction) with $750 \mathrm{~m}$ at nadir, 5 imaging (I) resolution bands (I1-I5; each has 32 detectors) with $375 \mathrm{~m}$ at nadir, and one panchromatic Day-Night-Band (DNB; 16 effective detectors) with $750 \mathrm{~m}$ throughout the Earth view swath. They are located on three different focal plane assemblies (FPAs): VIS/NIR, S/MWIR, and LWIR. The VIS/NIR focal plane is not temperature controlled, while the other two cold FPAs are maintained nominally at about $80 \mathrm{~K}$ by a passive radiative cooler assembly.

VIIRS carries a set of on-board calibrators (OBCs) for onorbit calibration. They are the solar diffuser (SD) and the solar diffuser stability monitor (SDSM) for reflective solar bands (RSB; bands M1-M11, I1-I3, and DNB) calibration, and blackbody (BB) for thermal emissive bands (TEB; bands M12-M16 and I4-I5) calibration. It also has a deep space view (SV) designed to allow background signal measurement scan by scan. The SV port can be used for lunar observations to provide relative calibration to improve $\mathrm{SD} / \mathrm{SDSM}$ measurements [1]. VIIRS consists of 22 spectral bands covering from $412 \mathrm{~nm}$ to $12 \mu \mathrm{m}$. Among the spectral bands, 7 of them are TEB infrared mid- and long-wave channels. Table 1 lists the thermal bands specified wavelength and typical scene brightness temperature along with the specified noise equivalent differential temperature (NEdT). Noted that M13 is a dual gain band operating on gain stage either at high or low. The TEB are basically calibrated on-orbit by the BB and SV on a scan-by-scan basis using a quadratic algorithm that converts detector's response to TOA radiance from the nadir observation. The coefficients and parameters used in the L1B calibrations are traceable to pre-launch measurements.

This paper describes the TOA TEB calibration uncertainty algorithm used in the L1B data processing by NASA SIPS. 
It covers the radiometric uncertainty but does not cover any geometric uncertainty caused by band-to-band and detectorto-detector spatial registration errors.

Table 1. VIIRS thermal emissive bands specification.

\begin{tabular}{|c|c|c|c|c|c|c|c|c|}
\hline Band & 14 & M12 & M13Hi & M13Lo & M14 & M15 & 15 & M16 \\
\hline$\lambda(\mu \mathrm{m})$ & 3.74 & 3.70 & 4.05 & 4.05 & 8.55 & 10.763 & 11.45 & 12.013 \\
\hline$T_{\text {typic }}(\mathrm{K})$ & 270 & 270 & 300 & 380 & 270 & 300 & 210 & 300 \\
\hline NEdT (K) & 2.5 & 0.396 & 0.107 & 0.423 & 0.091 & 0.07 & 1.5 & 0.072 \\
\hline
\end{tabular}

\section{TEB ON-ORBIT CALIBRATION ALGORITHM}

On orbit, the TEB are calibrated by an on-board calibrator BB controlled at a fixed temperature $\sim 292.5 \mathrm{~K}$, which is monitored by 6 thermistors every scan. Below is the calibration equation of the Earth View (EV) TEB spectral radiance (L) entering the instrument nadir aperture (ap) described in the VIIRS Algorithm Theoretical Basis Document (ATBD) [2]:

$L_{E V_{-} a p}=\frac{\left(R V S_{S V}-R V S_{E V}\right)}{R V S_{E V}} \cdot\left\{\begin{array}{l}{\left[\frac{\left(1-\rho_{R T A}\right) L_{R T A}-L_{H A M}}{\rho_{R T A}}\right]+} \\ F_{f a c} \cdot\left(c_{0}+c_{1} \cdot d n_{E V}+c_{2} \cdot d n_{E V}^{2}\right)\end{array}\right\}$

where

RVS: response versus scan angle of the HAM $\rho$ : rotating telescope assembly mirror reflectance $\mathrm{c}_{\mathrm{i}}$ : calibration coefficients measured pre-launch dn: digital number response with SV background subtracted

TEB EV radiance retrievals are computed scan-by-scan for each band, detector, HAM side, and gain stage (M13 only) in L1B processing software. The calibration F-factor $\mathrm{F}_{\text {fac }}$ is calculated each scan using BB modeled radiance, including the self-emission from RTA and HAM, over the observed radiance:

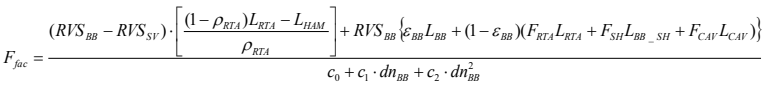

Overall, the S-NPP VIIRS on-board BB has been extremely stable. Figure 1 shows the BB average temperature from 6 thermistors during the nominal operation. There are three periods of time in the beginning of mission that $\mathrm{BB}$ has a little higher temperature due to different settings. The TEB detectors have been performing very well with small but steady changes, and the behaviors are consistent across all the detectors and both HAM sides in each band [3]. Figure 2 shows TEB 5-year band average F-factor trending since launch. Most TEB detectors are steady except for band I5 which has largest increase about $15 \%$.

On a quarterly basis, the BB performs warm-up and cooldown (WUCD) operation varying the temperature between ambient $(267 \mathrm{~K})$ and $315 \mathrm{~K}$ to monitor the on-orbit changes in calibration quadratic coefficients $c_{i}$. It takes about 46 hours to complete a WUCD cycle each time. Figure 3 plots band M14 detector response digital numbers (dn) of all 16 detectors (HAM side A only) during the cooldown period, which produce calibration fitting coefficients. These data at different $\mathrm{BB}$ temperatures are also examined to monitor any change of detector's noise NEdT at different scene radiance levels throughout the mission as shown in Figure 4 for band M12. The WUCD data sets are useful for error analysis and being applied for uncertainty estimate.

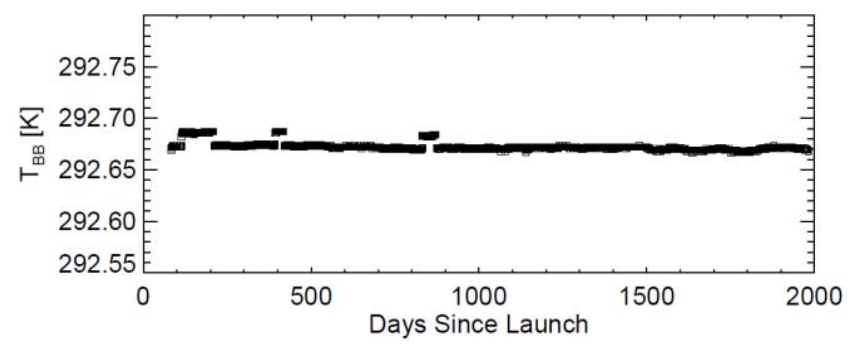

Figure 1. On-board BB temperature trending at nominal setting.

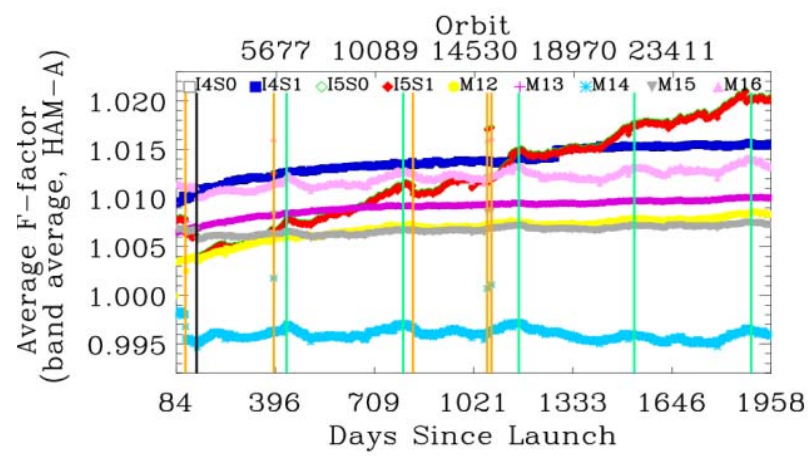

Figure 2. S-NPP VIIRS TEB band average F-factor trending.

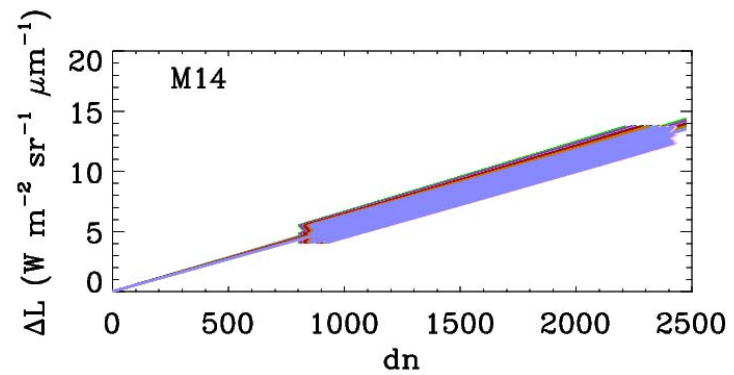

Figure 3. TEB M14 responses dn (all detectors, HAM-A) versus BB modeled radiance during BB cool-down (Sep 2016).

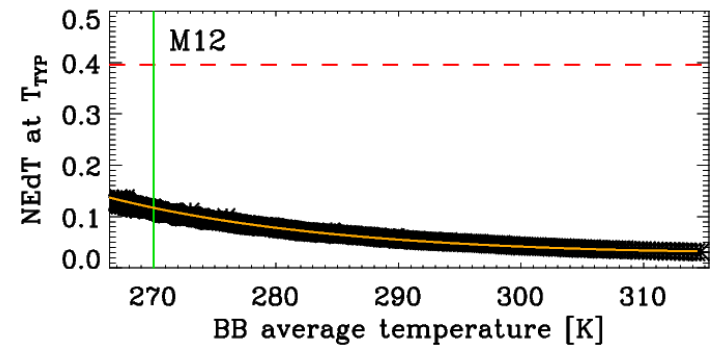

Figure 4. TEB M12 NEdT during BB cool-down (Sep 2016). 


\section{RADIOMETRIC CALIBRATION UNCERTAINTY}

The NASA VIIRS SIPS and VCST have worked together and developed VIIRS Level-1 Software Suite. This software produces Level-1A (L1A) and L1B products and is distributed by SIPS for the Atmosphere, Land, and Ocean disciplines. The first L1B software version V1.1 was released in January 2016 followed by V2.0 update in October 2016. Since then, the SIPS science disciplines have been running the L1B software to process and reprocess the VIIRS data using L1A input, generated from the L1A software developed by the Ocean SIPS, and the calibration Look-Up-Tables (LUTs) provided by VCST. However, the current VIIRS L1Bs do not have uncertainty product like MODIS. The radiometric calibration uncertainty algorithms and product design for RSB, TEB, and DNB are being developed by VCST and to be implemented in the future L1B software and products. The TEB uncertainty algorithm is based on the on-board blackbody calibration and scene radiance retrieval equations (1) and (2) described above. The retrieved EV scene radiance is a function of pre-launch determined parameters and on-orbit measured variables:

$$
L_{E V}=L_{E V}\left(\begin{array}{l}
c_{0}, c_{1}, c_{2}, R V S_{S V}, R V S_{B B}, R V S_{E V}, \varepsilon_{B B}, \rho_{R T A}, \lambda, T_{B B}, \\
T_{H A M}, T_{R T A}, T_{S H}, T_{C A V}, F_{R T A}, F_{S H}, F_{C A V}, d n_{B B}, d n_{E V}
\end{array}\right)
$$

Equation (3) lists all the contributors to uncertainty in the EV radiance retrieval for each pixel. Instead of deriving a complex error propagation equation using individual parameters with partial derivatives, we can estimate the uncertainty by neglecting the error contributions from the second and higher orders. Thus the error propagation equation can be reduced to less complex terms dominated by the first order. Using a small perturbation approach used by NASA MODIS L1B's error analysis [4], the EV total uncertainty can be described approximately as:

$$
\left(\frac{d L_{E V}}{L_{E V}}\right)^{2} \equiv \sum_{i}\left(\frac{L_{E V}\left(x_{i}+d x_{i}\right)-L_{E V}\left(x_{i}\right)}{L_{E V}\left(x_{i}\right)}\right)^{2}
$$

For those parameters which are determined pre-launch, their error budget can be assessed through the calibration tests on the ground either during the ambient environment or thermal vacuum [5]. They are HAM RVS, BB emissivity, RTA mirror reflectance, wavelength, temperature thermistor accuracy, and cavity geometry factors. These error budgets are estimated pre-launch and won't be changed unless evidence shows these parameters have changed post launch.

For the variables that can be measured on orbit, including radiometric fitting coefficients, temperatures, and digital numbers of $\mathrm{BB}$ and $\mathrm{EV}$, their error budgets are timedependent and will be calculated in L1B software scan-byscan or by the L1B uncertainty LUT update as needed. It was described earlier that the quadratic coefficients are monitored quarterly. Because the coefficient trends are stable and comparable to pre-launch values, they have not been updated post-launch [1] [3]. The uncertainty in the HAM temperature is estimated at $\pm 1 \mathrm{~K}$ while the cavity and BB shield temperature uncertainty are estimated at $\pm 2 \mathrm{~K}$. The uncertainty for RTA temperature is higher at $\pm 4 \mathrm{~K}$ because there is no direct measurement but estimated from surrounding cavity temperatures and thermal model [5]. The BB temperature uncertainty is estimated by the standard deviation of $6 \mathrm{BB}$ thermistors' temperatures and will be used in total uncertainty calculation.

During WUCD process, the BB is controlled at different fixed temperatures for more than one orbit at each temperature plateau. Such data are used for thermal analysis. However, the uniformity of $\mathrm{BB}$ exceeds the specification of $30 \mathrm{mK}$ when it is transitioning from one temperature plateau to another, as shown in Figure 5 (b). The periodic pattern is due to orbital variation. The impact to the L1B retrieval can be seen by examining the behaviors of calibration coefficient F-factor. Figure 6 plots the MWIR dual gain band M13 F-factor of all 16 detectors (HAM-A only) over the period of WUCD. There is about $0.3 \%$ variation in the F-factor due to the $\mathrm{BB}$ set temperature transition. This indicates there are residual errors from the radiance retrievals including, possibly, pre-launch coefficients and parameters, telemetry, or some imperfection in radiometric ATBD [6].

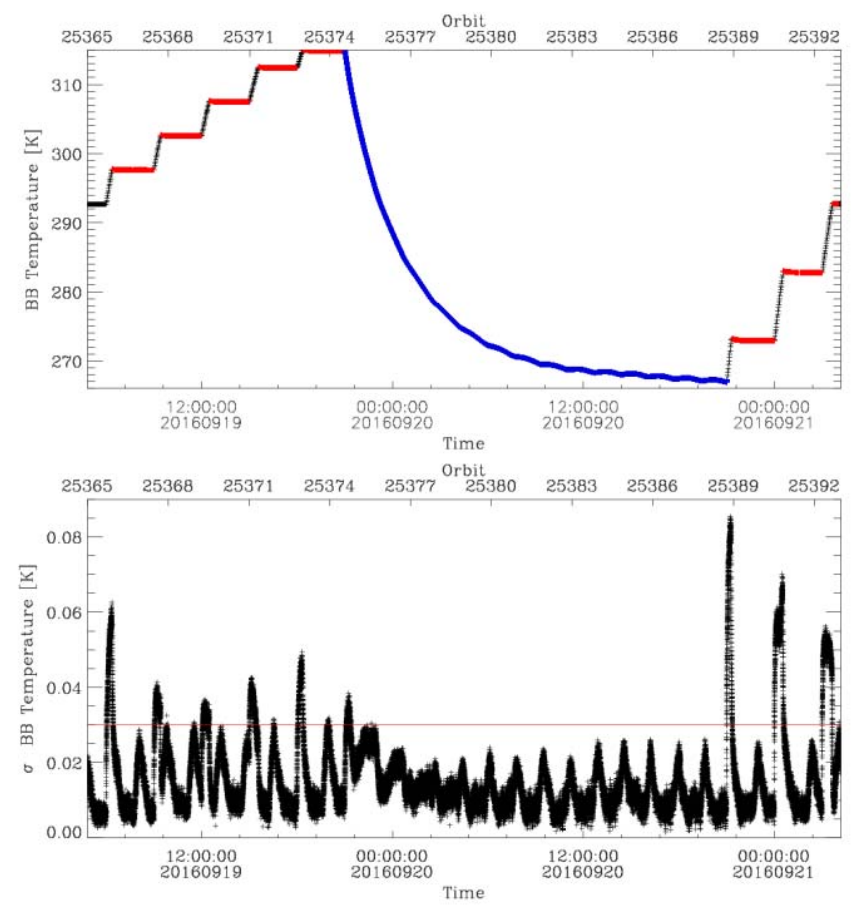

Figure 5. (a) Top: Blackbody temperature profile and (b) Bottom: BB thermal uniformity during warm-up cool-down (Sep 2016). The uniformity specification of $\mathrm{BB}$ is at $30 \mathrm{mK}$. 
It has been reported that the WUCD calibration activity yields unwanted spikes in the VIIRS derived Sea Surface Temperature (SST). The results impact the quality of the science and environmental data records related to the period of quarterly WUCD activities. The implementation of uncertainty algorithm in the L1B will provide the radiometric uncertainty of radiance in percentage for each calibrated pixel. It will help the data users understand the quality of the products as well.

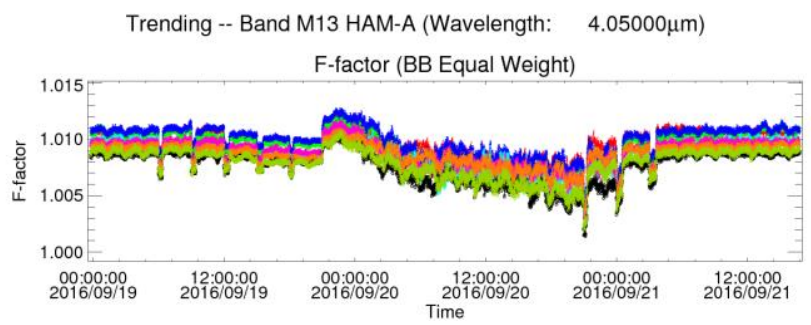

Figure 6. TEB M13 F-factor (all detectors, HAM-A) during BB WUCD period (Sep 2016).

\section{SUMMARY}

The method of estimating VIIRS TEB L1B radiometric calibration uncertainty has been described. All these error analysis allows us to better understand the behaviors of the sensor and estimate the calibration uncertainties associated with each component contributing to total TOA radiances. The implementation of uncertainty algorithm in the L1B software and the new uncertainty product will provide data quality information similar to those in MODIS products.

The authors would like to acknowledge Sergey Gusev and Yonghong Li of VCST for their help in data analysis and graphics to this work and continued support throughout the uncertainty analysis.

\section{REFERENCES}

[1] Xiong X., J. Butler, K. Chiang, B. Efremova, J. Fulbright, N. Lei, J. McIntire, H. Oudrari, J. Sun, Z. Wang, and A. Wu, "VIIRS On-orbit Calibration Methodology and Performance," JGR Vol. 119, Issue 9, pp 5065-5078, 2014.

[2] Joint Polar Satellite System (JPSS) Visible Infrared Radiometer Suite (VIIRS) Sensor Data Records (SDR) Algorithm Theoretical Basis Document (ATBD); Technical Report; Goddard Space Flight Center: Greenbelt, MD, USA, 2013.

[3] Efremova, B., J. McIntire, D. Moyer, A. Wu, and X. Xiong, "SNPP VIIRS thermal emissive bands on-orbit calibration and performance," J. Geophys. Res. Atmos., 10.1002/2014JD022078, 2014.
[4] Chiang, K., X. Xiong, A. Wu, and W. Barnes, "MODIS thermal emissive bands calibration uncertainty analysis," Proc. of SPIE Vol. 5542, doi: 10.1117/12.558072, 2004.

[5] Johnson, E., K. Galang, C. Ranshaw, and B. Robinson, "NPP Visible/Infrared Imager Radiometer Suite (VIIRS) Radiance Uncertainty, Emissive Bands - Tested Performance," Proc. of SPIE Vol. 7808, 78081F, 2010.

[6] Moyer, D., J. McIntire, F. DeLuccia, B. Efremova, K. Chiang, and X. Xiong, "VIIRS Thermal Emissive Bands Calibration Algorithm and On-orbit Performance," Proc. of SPIE Vol. 8510, doi: 10.1117/12.930145, 2012. 